\title{
IN MEMORIAM: JULIO RUIZ BERRIO Y FERRÁN FERRER
}

IN MEMORIAM: Julio Ruiz, Berrio and Ferrán Ferrer

\author{
Luis Miguel Lázaro *
}

Este año ha sido bien triste en el plano personal-institucional para todos los que formamos parte de la Sociedad Española de Educación Comparada. Con no muchos meses de diferencia han fallecido dos queridos colegas, compañeros y amigos acreedores ambos de todo nuestro cariño personal y respeto intelectual: Ferrán Ferrer Julià y Julio Ruiz Berrio, Catedráticos del área de Teoría e Historia de la Educación en la que se incardina nuestra área de docencia e investigación, en la Universidad Autónoma de Barcelona el primero y en la Universidad Complutense de Madrid el segundo. De la bonhomía, generosidad y buen hacer en todos los planos que les caracterizaba puedo dar testimonio personal. He tenido el privilegio de haber formado parte de la Junta Directiva de nuestra Sociedad, bajo la Presidencia de José Luis García Garrido, entre 1994-1999, como Vocal acompañando al profesor Ferrer Julià en el mismo puesto y al profesor Ruiz Berrio como Vicepresidente. Más tarde, entre 1999-2002, con Ángel González Hernández como Presidente, el profesor Ferrer será el Secretario, el profesor Ruiz trabajará como Vocal, y lo les acompañaré como Vicepresidente. Todo lo vivido y compartido con ellos en esos años enriquece y conforma mis recuerdos, y alimenta ahora el impulso institucional a rendirles el merecido tributo y homenaje que por su rica trayectoria personal y académica les debe la Sociedad Española de Educación Comparada.

La vinculación académica del profesor Ruiz Berrio con la Educación Comparada es bien temprana. De hecho, ya en el curso 1968-69 imparte la asignatura de "Educación

\footnotetext{
* Presidente de la Sociedad Española de Educación Comparada.

Universitat de València (España).
} 
Comparada" por primera vez en la Universidad Complutense de Madrid con carácter de optativa (Ferrer y Naya 2010: 135). Se encuentra en el núcleo de profesores que participa en el II Congreso Mundial de Educación Comparada en 1974 en Ginebra sobre "La eficacia y la ineficacia de las escuelas secundarias". También en el III Congreso Mundial y VIII Asamblea de la Comparative Education Society in Europe, CESE, celebrados de forma conjunta en Londres en 1977 con el tema "Unidad y diversidad mediante la educación" (Tusquets 1979: 127). En 1979 asiste y participa activamente en la IX Conferencia de la CESE celebrada en Valencia sobre "Influencias de la investigación educativa internacional en las políticas nacionales de educación” (Formentín 1980: 28). Institucionalmente, en 1980 es "consejero de la Comisión Ejecutiva" de la Sociedad Española de Pedagogía Comparada que preside Ricardo Marín. Obtenida su Cátedra de Historia de la Educación en la Universidad de Oviedo impulsa la creación del Centro de Pedagogía Comparada de la Universidad de Oviedo siendo su primer Presidente (BSEPC 1983: 25). Justo por esas fechas, 1985, edita Educación Comparada, como Diccionario de Ciencias de la Educación, obra de la que era coordinador. Ruiz Berrio daba un perfil habitual en otros Catedráticos de Educación Comparada españoles que también trabajaban e investigaban en Historia de la Educación. En esa línea es uno de los promotores de la creación de la Sección de Historia de la Educación de la Sociedad Española de Pedagogía, germen de la Sociedad Española de Historia de la Educación de la que será su primer presidente en 1989. Fue también el creador de la Sociedad Española para el Estudio del Patrimonio Histórico-Educativo en 2003. Sin embargo, no dejará de seguir colaborando en distintas actividades del área de Educación Comparada, además de con las ya citadas responsabilidades directivas en nuestra Sociedad en los años noventa, con la coordinación en 2000 de la obra La cultura escolar de Europa, tendencias históricas emergentes, donde se recogen trabajos que en una perspectiva visiblemente comparativa en procedencia, autores y perspectivas abordan un tema relevante en la Historia de la Educación como es el de la cultura escolar. O la excelente edición crítica en 2007 de una obra clásica de la Educación Comparada como La teoría de las corrientes educativas de Pedro Roselló Blanch.

Ferrán Ferrer Julià se forma en el ámbito de la Educación Comparada en la Universidad Autónoma de Barcelona, donde a comienzos de los años ochenta Josep $\mathrm{M}^{\mathrm{a}}$ Quintana Cabanas es el Director del Centro de Pedagogía Comparada de la Universidad Autónoma de Barcelona adscrito a la Sociedad Española de Pedagogía Comparada, que tiene además en funcionamiento un Seminario de Documentación de Pedagogía Comparada en relación con los materiales existentes en el área de Barcelona (Quintana 1983: 3). En 1983 Ferrán Ferrer es contratado como profesor Encargado de curso para impartir las asignaturas Estadística aplicada a la Educación y Educación Comparada (Educar 1983: 201), y colabora tanto en las tareas del citado Centro como en proyectos de investigación con otros profesores de la Sección de Ciencias de la Educación de su Universidad como Jaume Sarramona (Educar 1983: 196). Uno de sus primeros trabajos académicos -"El sistema educatiu de la U.R.S.S."-se publica precisamente en la revista Educar que en 1983 dicha 
Universidad dedica de forma monográfica a la Pedagogía Comparada. No es casual que ese texto esté escrito en catalán. Sin perder nunca el referente comparado internacional -Vgr., su trabajo de 1998 sobre el Informe Delors-, el compromiso y preocupación de nuestro colega con la realidad y transformación innovadora en una perspectiva de equidad de la situación educativa de Cataluña será una constante en su trayectoria profesional. Buen ejemplo de ello son sin duda las investigaciones que con el apoyo de la Fundació Jaume Bofill dirige sobre los resultados del PISA en Cataluña en 2003 y 2009 o su trabajo de 2008 L'estat de l'educació a Catalunya. Temática que ocupará su actividad investigadora en los últimos años, traducida en publicaciones sobre esos problemas en Revista de Educación (2006), Cuadernos de Pedagogía (2007), Revista Española de Pedagogía (2010), Foro de Educación (2011), o su tarea en la coordinación del monográfico de la Revista Española de Educación Comparada (2012) dedicado a "PISA: aportaciones e incidencia sobre las políticas educativas nacionales". Por otra parte, su preocupación epistemológica por nuestra disciplina es patente en obras como Educación Comparada: fundamentos teóricos, metodología y modelos, 1990, o La educación comparada actual de 2002, que han ayudado a la formación de muchos jóvenes investigadores en nuestro campo. Además de las responsabilidades institucionales con la SEEC ya citadas al comienzo de éste texto, al poco tiempo de la obtención de su Cátedra en la Universidad Autónoma de Barcelona pasa a presidir nuestra Sociedad entre 2002 y 2006. Una etapa que dedicará a consolidar los avances conseguidos en años anteriores al tiempo que impulsará nuevas iniciativas de reconocimiento a la tarea de investigación de nuestros jóvenes colegas, o a la utilización de las nuevas tecnologías en red para facilitar la comunicación en el terreno académico entre todos aquellos que trabajamos en el ámbito de la Educación Comparada e Internacional.

Sirvan estas breves e incompletas notas biográficas como reconocimiento y tributo inicial de nuestra Sociedad a ambos colegas a la espera de posteriores contribuciones más amplias, plurales y sosegadas de colegas que también han tenido el privilegio de conocer, trabajar y compartir amistad con Julio Ruiz Berrio y Ferrán Ferrer.

\section{REFERENCIAS BIBLIOGRÁFICAS}

- (1983): "Actividades y noticias de los Centros de la Sociedad Española de Pedagogía

Comparada", Boletín de la Sociedad Española de Pedagogía Comparada, 6, 21-26.

- (1983): “Activitats generals del professorat de la Secció", Educar, 3, 195-201.

FORMENTÍN, J. (1980): "Crónica de la IX Conferencia de la Sociedad Europea de

Educación Comparada”, Boletín de la Sociedad Española de Pedagogía Comparada, 3, pp. 12-34.

FERRER, F. (1990): Educación Comparada: fundamentos teóricos, metodología y modelos.

(Barcelona, PPU). 
FERRER, F. (1998): Cap a l'educació de l'any 2000: una visió de l'informe Delors (Barcelona, Servei de Publicacions de la Universitat Autònoma de Barcelona).

FERRER, F. (2002): La educación comparada actual (Barcelona, Ariel).

FERRER, F. (Dir.) (2006): PISA 2003 a Catalunya, una ullada a les desigualtats educatives, (Barcelona, Fundació Jaume Bofill).

FERRER, F. et al. (2008): L'estat de l'educació a Catalunya (Barcelona, Mediterrània).

FERRER, F. y NAYA, L. M. (2010): "La Enseñanza de la Educación Comparada en España. Historia y Presencia en los Nuevos Grados de Educación", Revista Iberoamericana sobre Calidad, Eficacia y Cambio en Educación, 2, pp. 133-147.

FERRER, F. (Dir.) (2011): PISA 2009: avaluació de les desigualtats educatives a Catalunya (Barcelona, Fundació Jaume Bofill).

QUINTANA CABANAS, J. M. (1983): “Presentación”, Educar, 3, pp. 3-5.

ROSSELLÓ BLANCH, P. (2007): La teoría de las corrientes educativas y otros ensayos, edición crítica e introducción de Julio Ruiz Berrio (Madrid, Biblioteca Nueva).

RUIZ BERRIO, J. (Coord.) (1985): "Educación Comparada", Diccionario de Ciencias de la Educación (Madrid, Anaya).

RUIZ BERRIO, J. (Ed.) (2000): La cultura escolar de Europa, tendencias históricas emergentes (Madrid, Biblioteca Nueva).

TUSQUETS, J. (1979): “La aportación española al comparativismo pedagógico”, Revista de Educación, 260, pp. 115-131. 